\title{
Fast Inverse Compositional Image Alignment with Missing Data and Re-weighting
}

Vincent Lui

vincent.lui@monash.edu

Dinesh Gamage

dinesh.gamage@monash.edu

Tom Drummond

tom.drummond@monash.edu
ARC Centre of Excellence for Robotic Vision, Monash University,

Clayton, Australia
Image alignment is often performed using variants of the Lucas-Kanade algorithm [3]. Among these variants, the inverse compositional (IC) [1] method and the efficient second-order minimization (ESM) [2] method are the most efficient variants. While ESM is computationally more efficient for 2D image alignment problems such as homographies, IC is more computationally efficient for image alignment problems using RGBD data where it is expensive to re-compute the Jacobian.

In this paper, we look at methods to accelerate the convergence of IC and ESM algorithms while remaining robust to outliers. We propose a preconditioning strategy to perform inverse composition with reweighting and missing data which avoids the need to re-compute the Jacobian and its Hessian at every iteration. We also consider how the effects of image noise and/or spectral aliasing over the scale of the deformation can be used to speed up the convergence of all these methods.

The inverse compositional Lucas-Kanade algorithm minimizes the following cost function:

$$
C_{\mathrm{IC}}=\sum_{\mathbf{x}}[T(\mathbf{M}(\mathbf{x} ; \Delta \mathbf{p}))-I(\mathbf{M}(\mathbf{x} ; \mathbf{p}))]^{2}
$$

with respect to $\Delta \mathbf{p}$. $\mathbf{p}$ is the parameter vector and $\mathbf{x}$ is the pixel location in the template. $\mathbf{M}(\mathbf{x} ; \mathbf{p}))$ is a warping function which maps the pixel $\mathbf{x}$ in the template $T$ to a sub-pixel location $I(\mathbf{M}(\mathbf{x} ; \mathbf{p}))$ in the reference image $I$. As $J_{\mathbf{X}}$ is computed using the template image $T$ which does not depend on $\mathbf{p}$, it can be pre-computed as $J(0)$. Equation (1) is minimized iteratively in a non-linear least squares manner. The parameter vector $\mathbf{p}$ is related to the error vector $\mathbf{e}$ as

$$
J \Delta \mathbf{p}=\mathbf{e} .
$$

where $J$ is the Jacobian. At each iteration, we form the normal equations

$$
H \Delta \mathbf{p}=J^{T} \mathbf{e},
$$

where $H=J^{T} J$ is the Hessian. $\Delta \mathbf{p}$ is then computed as $\Delta \mathbf{p}=H^{-1} J^{T} \mathbf{e}$. The re-weighted version of the problem can be posed as $\left(J^{T} W J\right) \Delta \mathbf{p}=$ $J^{T} W \mathbf{e}$, where $W$ is usually a diagonal matrix. Inverse composition with re-weighting requires the Jacobian and Hessian to be re-computed at every iteration.

Conventionally, the pseudo-inverse of the Hessian is computed using Cholesky factorization. Here, we propose to perform a QR factorization on the Jacobian $J$ instead. This allows us to re-formulate Equation (2) as

$$
Q\left[\begin{array}{c}
R \\
0
\end{array}\right] \Delta \mathbf{p}=\mathbf{e},
$$

where $Q$ is an $m \times m$ orthogonal matrix where $Q^{T}=Q^{-1}$, and $R$ is an $m \times n$ upper triangular matrix. The parameter updates $\Delta \mathbf{p}$ can now be computed as $\Delta \mathbf{p}=R^{-1} Q^{T} \mathbf{e}$. Without going into details here, the re-weighting problem can be formulated as

$$
\Delta \mathbf{p}=R^{-1}\left(Q^{T} W Q\right)^{-1} Q W \mathbf{e} .
$$

The factors $Q$ and $R$ can be pre-computed. This leaves the weighting matrix $W$ and the term $\left(Q^{T} W Q\right)^{-1}$ to be computed at every iteration. Here, we propose to use a preconditioning approach to approximate $\left(Q^{T} W Q\right)^{-1}$, where the goal is to construct a matrix $P$ such that the product $P^{-1}\left(Q^{T} W Q\right)$ has a smaller condition number to $Q^{T} W Q$. We propose three preconditioners, each with increasing complexity (see Figure 1). The first preconditioner is a scaled identity matrix where every element in the diagonal represents the inverse of the average weight. The second preconditioner is the Jacobi preconditioner, where the matrix $P$ is a diagonal matrix. Every element in the diagonal is computed by accumulating the sum of weighted, squared elements in the corresponding column of the

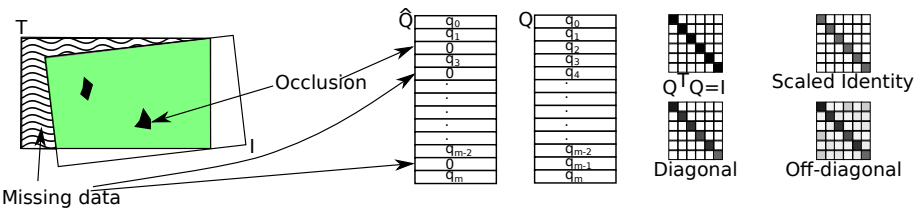

Figure 1: Left: Missing data and re-weighting causes the factor $Q$ to turn into a matrix $\hat{Q}$ that is no longer orthogonal. Right: The proposed preconditioners $P$. The product $P^{-1}\left(\hat{Q}^{T} \hat{Q}\right)$ should be as close to $I$ as possible. Here, a darker shade represents a value closer to 1.0.
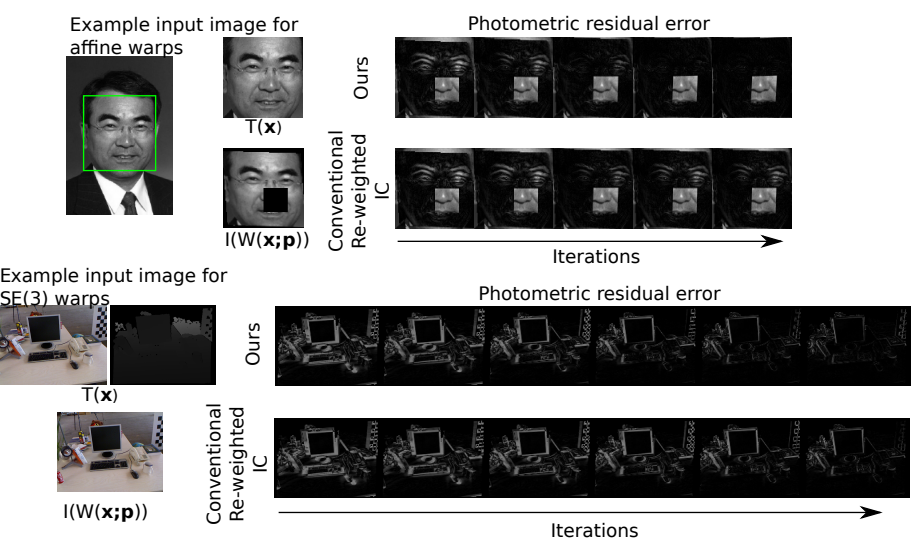

Figure 2: Qualitative results showing the how the residual photometric error changes with the number of iterations.

pre-computed factor $Q$. The last preconditioner extends the Jacobi preconditioner to accumulate off-diagonal elements as well. Assuming that the off-diagonal elements are close to zero, we approximate the inverse of this preconditioner using a first-order Neumann series.

Finally, we also investigated the effects of noise and/or spectral aliasing over the scale of deformation affects the convergence of image alignment algorithms. We found that noise and/or spectral aliasing acts as a regularizer term that damps the system and causes the parameter updates to be underestimated. We showed that a moderate increase in the step size of the parameter updates can further improve the convergence of image alignment algorithms.

We have evaluated our proposed methods using different motion models such as affine warps, homographies, and SE(3) warps (see Figure 2), and our experimental results show that our method is equally as robust as the conventional re-weighted inverse composition while exhibiting faster convergence rates. We found that the scaled identity and diagonal preconditioners offer the best trade-off between computational efficiency and robustness. For further details of our proposed method, including implementation details, experimental results, and a discussion, please refer to the full paper.

[1] S. Baker, R. Gross, T. Ishikawa, and I. Matthews. Lucas-Kanade 20 Years On : A Unifying Framework : Part 2. Technical report, 2003.

[2] S. Benhimane and E. Malis. Real-time image-based tracking of planes using Efficient Second-order Minimization. In International Conference on Intelligent Robot and Systems, volume 1, pages 943948, 2004.

[3] B.D. Lucas and T. Kanade. An iterative image registration technique with an application to stereo vision. In Proc. International Joint Conference on Artificial Intelligence, pages 674-679, 1981. 